\title{
Nurse-led research capacity building: developing a local strategy with direct- care rehabilitation nurses
}

\section{Letitia Burridge* PhD, MPH, BN(Hons)}

Research Fellow, The Hopkins Centre: Research for Rehabilitation and Resilience, Menzies Health Institute Queensland, Griffith University, Brisbane, QLD

Postal address: Division of Rehabilitation, Room 1SI.32.4, Level 1, Building 17, Princess Alexandra Hospital, Ipswich Road, Woolloongabba, OLD 4102

Email l.burridge@griffith.edu.au

\author{
Alison New MCR, MHSc (CE), BHScN \\ Nurse Educator (Brain Injury Rehabilitation), Brain Injury Rehabilitation Unit, Princess Alexandra Hospital \\ Ipswich Road, Woolloongabba, QLD 4102
}

David Lewis MN (Care of Older Person), GradCertHlthMgt, DipHlthSc (Nursing)

Nurse Unit Manager, Bunya Place, Geriatric and Rehabilitation Unit, Princess Alexandra Hospital, Ipswich Road, Woolloongabba, QLD 4102

Jenny Kohlhardt M. Ed, MN, BN

Clinical Nurse Consultant (CNC) Training; Education; Leadership

Princess Alexandra Hospital, Ipswich Road, Woolloongabba, QLD 4102

\section{Tracey Tattam Grad Cert HPE}

Nurse Educator, Spinal Injuries Unit, Princess Alexandra Hospital, Ipswich Road, Woolloongabba, QLD 4102

${ }^{*}$ Corresponding author

Keywords rehabilitation nursing, clinical nursing research, program development, research capacity building

For referencing Burridge $L$ et al. Nurse-led research capacity building: Developing a local strategy with direct-care rehabilitation nurses. Journal of the Australasian Rehabilitation Nurses' Association 2020; 23(2):17-24.

DOI https://doi.org/10.33235/jarna.23.2.17-24

\section{Abstract}

Introduction Direct-care rehabilitation nurses often want to lead research but lack the skills and resources. Many research capacity building $(\mathrm{RCB})$ models have been used and evaluated, yet the literature is thin regarding the early steps of program development for direct-care nurses.

Aim This paper describes one team's experience of the process of developing a RCB program.

Method This paper explains steps in the developmental process of a program of RCB for direct-care rehabilitation nurses. The process is framed by a specific theoretical model and the practical, contextual assumptions which had to be taken into account.

Results The two overarching conceptual components in the developmental process were determining objectives, and identifying and resolving risks. Steps in the process are outlined, together with how risks were managed while progressing toward the goal: the inaugural Rehabilitation and Research Masterclass Program - Nursing (RaMP-N). The Program is briefly outlined. 
Discussion This paper fills a gap by describing the formative stage of the RaMP-N that was crucial to its quality and relevance. The experience upskilled and energised the team, and generated small nurse-led research projects. The program format and content remained flexible and tailored, consistent with adult learning principles and rehabilitation nursing. Further research is needed to test the transferability and sustainability of the RaMP-N.

Conclusion The RaMP-N program strengthened rehabilitation nurses' engagement with research. Conversations, consideration, consultation, commitment and collaboration underpinned the evolving program as strategies and modifications were identified, applied and evaluated. The process enhanced the program team's leadership skills and participants' research skills.

\section{Introduction}

Teaching hospitals fill an important role in generating high quality research to inform evidence-based practice. However, research engagement is challenging for clinicians, including directcare nurses whose patient-first focus and minimal offline time often leaves limited opportunity for non-clinical activities such as research (Brauer, Haines, \& Bew, 2007; Paget, Lilischkis, Morrow, \& Caldwell, 2014). This may be compounded by nurses' limited research knowledge, interest or motivation (McKee, Codd, Dempsey, Gallagher, \& Comiskey, 2017). Consequently, involvement in practice-based research as a regular aspect of professional nursing practice is difficult. By implication, this deprives the clinical and learning environment of the unique benefits of research that is informed by day-to-day clinical issues through a nursing lens.

A review of research capacity building (RCB) activity among clinical nurses confirms the need for RCB competence and culture, integration with vertical and horizontal structures, and RCB champions (Lode, Sørensen, Salmela, A-L, \& Severinsson, 2015). In other words, RCB cannot succeed without research competence to enhance quality in care. Further, RCB success depends on strategies to enhance a research culture and to implement findings in practice settings (Lode et al., 2015). The sustainability of RCB also requires organisational support so that infrastructure enables access to RCB resources, teamwork and networking, including opportunities for multidisciplinary research activity (Lode et al., 2015). The achievement of these goals among nurses requires nursing leadership that is strong, strategic and visible (Lode et al., 2015). RCB initiatives that consider these issues are seen as an essential part of the solution.

RCB is an ongoing multi-level process empowering individuals, teams and institutions to develop, conduct and report high quality research to address identified health challenges (Kahwa, Edwards, \& Mortley, 2016). RCB is underpinned by six principles (Box 1) widely found to be useful in diverse settings (Gee \&
Box 1. Principles of research capacity building (Gee \& Cooke 2018)

1. Promoting actionable dissemination

2. Developing research close-to-practice

3. Developing a support infrastructure

4. Supporting linkages and collaborations

5. Developing research skills and confidence in the health services workforce

6. Planning sustainability

Cooke, 2018). By implication, a successful RCB program requires the full suite of these principles to be in place. Neither the principles nor the implication are contested here. The point we stress here is that an approach incorporating big-picture principles need not preclude small beginnings.

Diverse approaches to development and implementation of nursing RCB models include those that involve linkages to universities with a formal training program and a funded academic consultancy (Chapman, Duggan, \& Combs, 2011; English, 2016; Larson, Cohen, Gebbie, Clock, \& Saiman, 2011). Other models operate within formal funding packages (McKee et al., 2017; Trytten, Wale, Hayes, \& Holmes, 2019). The establishment of formal resourcing recognises that RCB is not a simple undertaking. Motivated direct-care nurses with limited resources and experience may be deterred from developing a $\mathrm{RCB}$ program with limited funding, infrastructure and expertise. A bottom-up approach may be useful in these circumstances.

Another consideration is uncertainty whether an existing RCB model will work in a new context with minimal loss of fidelity, relevance or effectiveness. Although a 'final' model may be proven as best, it may be difficult to achieve elsewhere with the same level of success, and result in unexpected or disappointing outcomes. Further, implementing proven interventions can be problematic in environments that not only differ but also change over time (Greenhalgh, MacFarlane, Barton-Sweeney, \& Woodard, 2012). There is value in models that deliberately retain changeability so they can be tailored for variable and changing needs. Local 
strategies can support the local development of RCB activities that fit local conditions (O'Byrne \& Smith, 2011). Thus, a local and flexible approach to RCB can build on knowledge of the setting and accommodate contextual idiosyncrasies.

Assuming that an RCB intervention can progress from small beginnings, what are the practical steps involved, and what kind of trajectory should nurses expect as they develop an RCB intervention? More discussion is needed about what happens in the early scoping process of developing a RCB program for direct-care nurses. This is a bottom-up process that occurs in the presence of competing clinical priorities and limited resources. The formative period between the conception of a vision for a RCB program and its realisation is pivotal. It can mean the difference between pushing forward or giving up, yet the practical steps involved are rarely documented comprehensively.

The aim of this paper is to describe steps taken in the developmental process that culminated in a nurse-led, locally designed RCB program for direct-care rehabilitation nurses. Development of this RCB program was found to be a dynamic rather than linear process. The program went through recurring iterations and revisions to achieve an optimal fit with participants' RCB needs. By shedding light on this early process, other directcare rehabilitation nurses may be encouraged to see possibilities for investing themselves in local RCB initiatives to support nurseled research.

\section{Theoretical approach to the developmental process}

The value of a theoretical approach lies in its usefulness to explain how and why things work, how they interconnect, and how to make sense of situations (Germov, 2005). It was therefore a key task to identify a theoretical approach for understanding the steps in the developmental process of the RCB program. In the model of continuing professional development (CPD) Haywood, Pain, Ryan and Adams (2012), the unidirectional arrows suggest linearity in CPD activities, such as RCB, progressing forward from conception to maturity. Another framework for evaluating RCB (Cooke, 2005) describes the structural landscape and principles on which a RCB program might develop, but the dynamic and iterative nature of the developmental process is not explicit. Neither of these models explain the experience of the program team reported here.

In contrast, the Spiral Life-Cycle model (Boehm, 1988) illustrates program development as an iterative and evolving process.
This model originated in software development, where it has been used to acknowledge and depict the process of evolving cycles as project requirements are clarified and refined during development (O'Regan, 2018). The Spiral Life-Cycle is a dynamic model comprising four quadrants of key activities that start small while thinking big (Figure 1); these are determining objectives, identifying and resolving risks, development and testing, and planning the next iteration (Ruparelia, 2010). All four quadrants are critical to the cycle. The program team experienced a similar evolving process of planning, evaluating and modifying the program. Also consistent with a spiral diagram, the starting and finishing points of each loop of the developmental process were in different places as the program continued to evolve. These parallels between the Spiral Life-Cycle model and the evolution of the RCB program model in the study setting suggested it would be helpful for explaining the developmental process of the program.

\section{The developmental process}

Program development can be a cyclical and iterative process requiring agility and resolve to best meet the purpose of the program. The activities associated with determining objectives and with identifying and managing risks precede program delivery and subsequent planning in the process which then leads to the next iteration of the program. The upper two quadrants of Figure 1 comprise the early evolution of a program from the point of conception to the point when it is delivered. Describing the practical steps in the development of the Rehabilitation and Research Masterclass Program - Nursing (RaMP-N) may be useful to other direct-care rehabilitation nurses who are interested to develop a site-specific RCB program.

\section{Overview of the inaugural RaMP-N}

The first workshop of the inaugural program (2018) comprised a foundation 6-hour workshop including research basics, practicalities and planning. Six months later, a 6-hour advanced workshop was conducted comprising participant presentations, group interaction and feedback. Participants had access to mentoring during and between workshops.

In the first year of the program, the workshop size was intentionally capped at 12 participants. This was to optimise the tailored support that would enable participants to set and pursue the achievable milestones they adopted such as a literature review, a researchable question or a draft research protocol. For the inaugural program, participants had been purposively invited on the basis of their motivation to lead research. The rationale for this was that it was considered strategically important to 


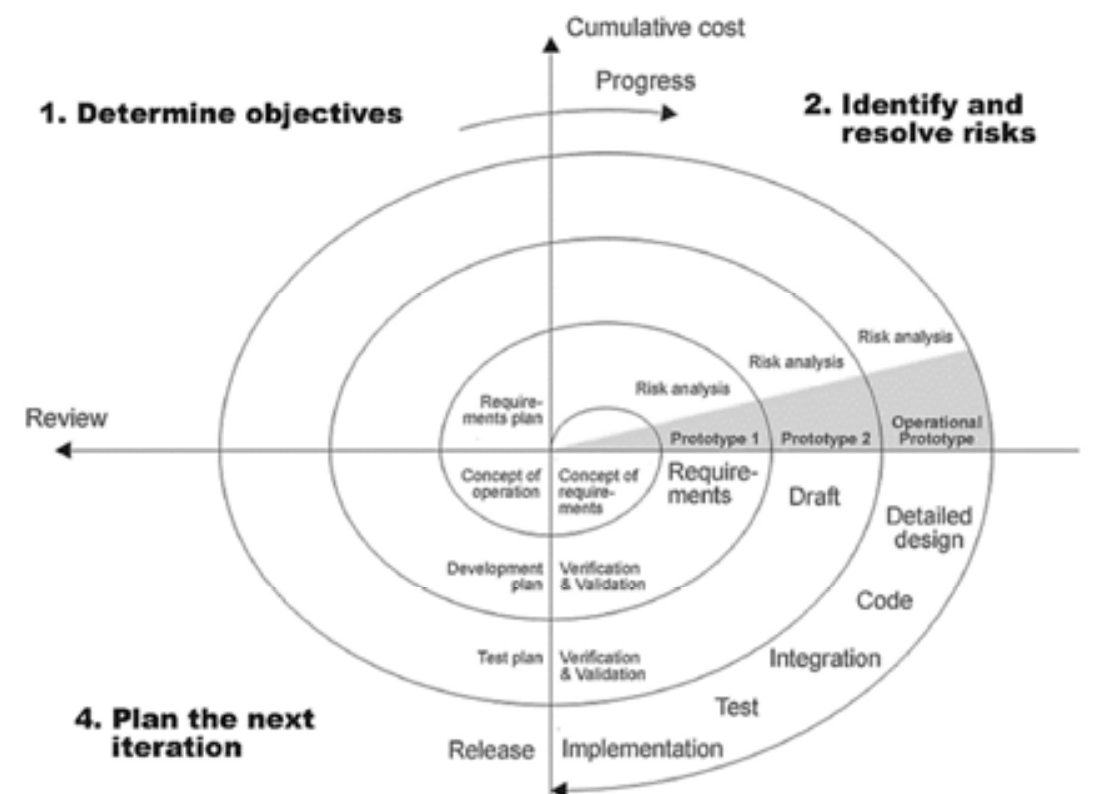

3. Development and Test

Figure 1. The Spiral Life-Cycle model (Boehm, 1988)

be able to demonstrate value through evidence of outputs and ongoing research-related activity among alumni participants. The program was designed to be consistent with principles of adult education (Kroehnert, 2010), so the workshops were interactive, tailored, flexible, collegial and co-creative, with an active mentoring component. Workshop I (foundation) drew upon expert presenters including librarians, research fellows and senior clinical nurses. The relaxed approach was conducive to questions and discussion. Workshop II (Advanced) mostly comprised participant presentations and feedback. An overview of the program is presented in Box 2.

\section{Steps taken in the developmental process, and the outcomes}

This section describes the two major components of the developmental process of the RaMP-N program, with reference to the upper two quadrants of Figure 1, determining objectives, and identifying and managing risks. These steps are listed in Box 3 and discussed in detail below.

\section{Determining objectives}

Determining the objectives entailed several key interrelated steps - understanding the context, shaping the vision, and clarifying the purpose, aim and objectives of the program.

\section{Context}

The team defined the context for the RaMP-N. Quality improvement (QI) activities or recruitment and data collection for
Box 2. Overview of the first iteration of the RaMP-N

Workshop I (Foundation) $\quad$ Workshop II (Advanced)

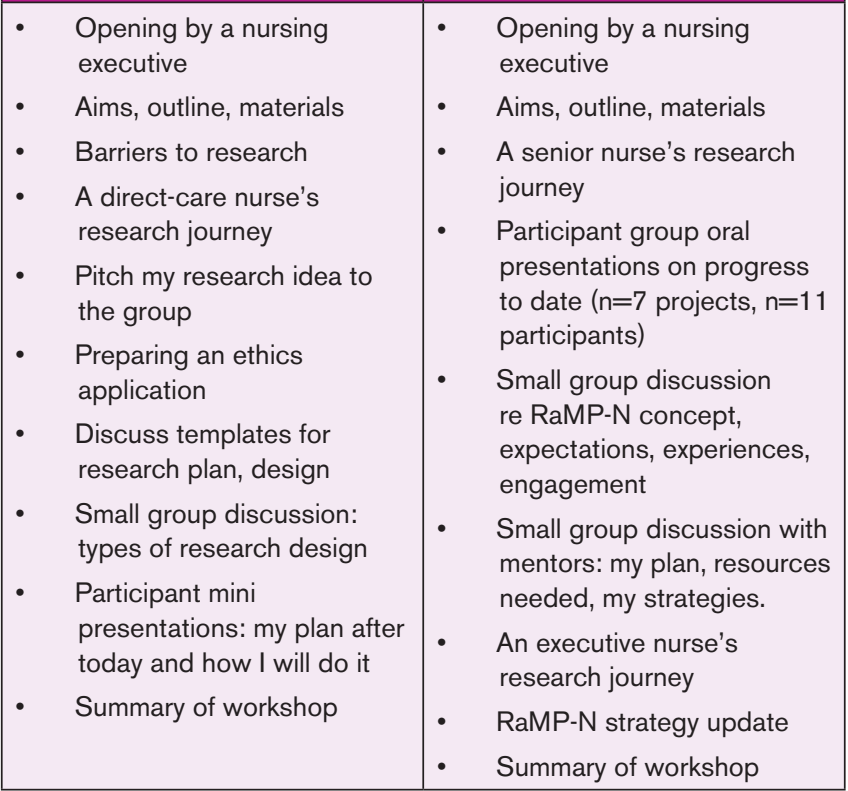

research projects led by other health professionals were typical in the study setting, regardless of nurses' level of education or research experience. Nurses in the study setting appeared to be less research-active and less engaged with research compared to other disciplines. It was apparent that the practice environment did not readily legitimise nursing research due to the round-theclock priority of direct-care, as others have found (Berthelsen, 
2020; Hagan, 2018; Marshall et al., 2016). Anecdotal evidence in the form of health professionals' comments in the study setting had suggested general misconceptions of rehabilitation nursing that belied the specialised nature of the role. This is consistent with more recent research (Digby, Bolster, Perta, \& Bucknall, 2018) confirming that non-nurses in rehabilitation settings tended to regard nurses as a support for therapists rather than as equal members of the multidisciplinary team.

Vision

The team had a long-held aspiration to increase the visibility of rehabilitation nursing and showcase the specialised nature of the practice and how it uniquely value-adds to the quality of rehabilitation services. The vision was fuelled by a professional self-belief regarding the unique value of rehabilitation nursing specialties, and by their everyday familiarity with individual directcare rehabilitation nurses' desire to conduct research. They had a passion to enable RCB in their workplace settings in parallel with their institutional priorities, opportunities and constraints.

\section{Purpose}

The team scoped the RaMP-N as a strategic organisational initiative to strengthen practice-based, nurse-led research. This would be achieved by increasing rehabilitation nurses' knowledge of research methods and by supporting them to develop and progress a research topic of their choice.

\section{Aim}

The aim of the program was to build direct-care rehabilitation nurses' capacity to scale up their clinical questions into research projects, and to equip and support them to lead their research projects.

\section{Objectives}

The objectives were to create a structured training and support program to target rehabilitation nurses' RCB needs, to identify nurses who were interested in gaining research knowledge, to deliver the program, and to provide mentoring support that was relevant, realistic and sustainable. The team liaised with nurse managers in each rehabilitation setting to identify potential participants on the basis of demonstrated interest in research linked directly to clinical practice. Invitations were sent by email and followed up in-person to ensure the invitees understood the nature of this training opportunity for themselves and its significance for the future development of rehabilitation nursing research within the study setting.

\section{Identifying and managing risks}

The second major component of the developmental process was to identify and manage risks. This also entailed several key interrelated steps - conversations, consideration, consultation, commitment and collaboration. These steps enabled the team to pace the developmental process so that risks and risk management strategies could be pre-identified as much as possible. For example, what might weaken participants' engagement with the program? What if a high quality facilitator is unavailable? Averting undesirable 'what-ifs' or managing them promptly would help optimise the desirable impact of the program.

\section{Conversations}

The initial conversations had started small and occasional. The RaMP-N was conceived in ad hoc face-to-face discussions and e-conversations among four like-minded senior rehabilitation nurses practising in different rehabilitation units at one site. In these encounters, they discussed their experiences, observations and interactions with direct-care rehabilitation nurses around the opportunity to build nurses' research capacity. Working as a team, the nurses recognised the tension between RCB opportunities and the priorities of clinical practice, and the potential risks related to program attendance and completion. Although informal, these motivated and ongoing conversations helped to preserve the vision and had a consolidating effect on what was becoming a highly functional team. Ideas were exchanged and discussed over a 12-month period, culminating on International Nurses' Day 2017 when the group resolved to stop talking about the RaMP-N and start planning it.

\section{Consideration}

As nurses, the team were accustomed to being reflexive in practice as a means of identifying and resolving challenges. This form of dialogue was also active in the context of RCB, as team members explored how to manage the challenges that seemed to impede their ambitious RCB goal. As ordinary as it may seem to nurses, this reflexivity was important because it acknowledged the opportunity to innovate while value-adding to and reinforcing individual and team capacity. It became apparent that the team would benefit from the expertise of others, including nursing executive.

\section{Consultation}

The team moved into consultation mode and briefed an on-site nursing executive whose rehabilitation specialty background made her very supportive although not clinically research- 
active. The nursing executive responded with sound strategic advice that encouraged and challenged the team to: (a) start by focusing on the process rather than the outcome; (b) take into consideration the strategic plans of the service; (c) consult with other executive nurses and divisional nurse leaders; (d) submit a formal application for a dedicated rehabilitation research nursing position; (e) think multi- rather than single-site; and ( $f$ ) keep nursing executive updated regarding progress. These tasks would demonstrate that the team were not only committed to the success of the RaMP-N, but were also capable, flexible, clear and undaunted regarding mutual expectations. They were also open to all options in moving forward - including unfunded options.

\section{Commitment}

The increasing complexity during the planning phase posed a risk to the progress toward an RCB program. Understanding risk was integral to the process of developing a sustainable program. Doing nothing would involve risk, because specialist nurses' capacity to improve the quality of rehabilitation services through nurse-led research would remain limited and their contribution to rehabilitation would remain under-recognised. However, developing the RaMP-N involved multi-level risks. For example, the program would need to be acceptable to nurses practising in direct-care as well as to management in order to build credibility around its potential and to embed its legitimacy within multidisciplinary rehabilitation. Further, the boundarycrossing nature of rehabilitation services created structural risks. The team would need to approach other clinical divisions for buyin that would optimise program promotion and participation. The innovation would be unfunded. Without a senior rehabilitation nurse researcher position in the organisation, the team was deliberating over a RCB strategy without the benefits of a local nurse champion.

\section{Collaboration}

This led to concern as to whether the vision was beyond reach without research expertise. However, in 2015 the Division of Rehabilitation at Princess Alexandra Hospital (PAH) in Queensland, Australia, became a founding partner of The Hopkins Centre (https://www.hopkinscentre.edu.au/). The Centre focuses on interdisciplinary applied research that examines rehabilitation practices, disability services and social support systems. The Centre also focuses on interdisciplinary rehabilitation research involving service users, clinicians and researchers to promote improvements in recovery and resilience (https://www.hopkinscentre.edu.au/). This partnership made it possible to progress the vision.
Through The Hopkins Centre, the team were able to draw on the research capacity and opportunity afforded by the rehabilitation services linked with the Centre. The partnership with the Centre emphasised an embedded rather than university-based model. This provided the team with access to guidance, encouragement and support from experienced research academics. One academic (LB) was co-opted onto the team in a collaborative capacity and provided or brokered support as needed. Planning developed steadily over the next 6 months, culminating in a program that was ready to operationalise. The RaMP-N then progressed through stages 3 and 4 of Figure 1 in 2018, and the cycle recommenced with the 2019 iteration of the program.

The RCB model has continued to be labile rather than mature into a final fixed state. The steps that were integral to the two preliminary stages (Box 3 ) remained active in the ongoing cycles of the RaMP-N. Determining objectives occurs within the current context, and is underpinned by a clear vision, purpose and aim. Identifying and managing risks is underpinned by a process of conversations, ongoing consideration and consultation, commitment and collaboration. Together, these steps have been fundamental to planning further iterations of the program.

Box 3. Steps in developing a RCB program for practising nurses

\begin{tabular}{|l|l|}
\hline 1. Determining objectives & 2. Identifying and resolving risks \\
\hline Define context & Conversations \\
Clarify vision & Consideration \\
Confirm purpose & Consultation \\
Establish aim & Commitment \\
Set objectives & Collaboration \\
\hline
\end{tabular}

\section{Discussion}

This paper sheds light on steps taken during the critical formative stage between the conception and delivery of a RCB program for direct-care nurses. It was driven by a desire to improve the quality of care through RCB by creating an opportunity for directcare nurses to develop and lead clinically relevant research in their clinical units. A secondary purpose was to showcase specialist rehabilitation nursing in a discerning interdisciplinary environment. This might address an acknowledged need for better understanding and appreciation of how nurses contribute to rehabilitation practice (Digby et al., 2018). Anecdotal comments from rehabilitation nurses and nursing management suggest that this is occurring.

The RaMP-N benefited from executive endorsement and reciprocated with outcomes that are tangible expressions of its alignment with the organisation's mission and priorities, and with 
the Magnet ${ }^{\circledR}$ designated status of PAH (Queensland Health, 2020). This occurred through an innovative program to enhance nurse-led research to underpin safe and excellent patient care.

The preliminary work of developing the first-ever training program for nurse-led rehabilitation research in the study setting was complex and demanding. The process itself was an exercise in innovation, generating productive energy within the team and cohesive ongoing relationships with those involved in the consultation and collaboration steps. The program also generated a surprising level of interest among participants. Through their reflexive approach, the team was accumulating 'capital' for innovation and reinvesting it into the program, which is consistent with the findings of others (Schippers, West, \& Dawson, 2015).

The program was feasible with limited resources. The experience of developing the RaMP-N was like using an orienteering map in a process landmarked with RCB principles for reference. Through coordinated effort using accessible knowledge and resources, the destination was reached. In parallel with the evolution of the program, clinically experienced research novices upskilled and overcame uncertainty to achieve a shared vision. This was consistent with Kolb's experiential learning cycle (revised) that involves contextually rich concrete experience, critical reflective observation, contextual-specific abstract conceptualisation, and pragmatic active experimentation (Morris, 2019). Rather than stalling without the full complement of RCB principles for success, the team learned how to innovate and how doing so enabled this small-scale program to happen.

The evolving RaMP-N model signified its customised nature rather than underdevelopment or instability. Instead of a standard, replicable model, the RaMP-N model normalised its adaptability for a stronger fit to the needs and expectations of changing participants and clinical contexts. This echoes the idea of agility in a living, breathing model that moves with change (Mundra, 2018). The team realised and understood this best after the second iteration of the program.

The experience of this team in the development of the RaMP-N made sense when considered in parallel with the agility of the Spiral-Life Cycle (Boehm, 1988) (Figure 1). They started small, considered risks, while looking ahead to explore future forms of the program. They expected to learn from, adjust to and accommodate changing needs with each iteration of the program. This model also legitimised the team's preference for a program tailored to changing needs in a dynamic clinical environment rather than a 'final' fixed model.

\section{Lessons learned}

There were three main lessons for the program team. Firstly, it was possible to operationalise the program with limited funding because it was complemented by a motivated team, access to onsite research expertise, and a supportive institutional environment. Secondly, the surprising level of interest among direct-care nurses reinforced the important distinction between research interest and research activity among rehabilitation nurses. Thirdly, some program alumni became research buddies and conduits to research expertise within their practice setting networks. These lessons would never have been learned without trying.

\section{Limitations}

The RaMP-N was intentionally tailored to local needs in local rehabilitation practice settings. The transferability of the steps as outlined to other settings is unknown. The sustainability and continuing evolution of the program is unclear. The 2020 program was not offered due to additional clinical pressures related to the COVID-19 pandemic. Planning for a 2021 program has commenced, including exploration of opportunities to re-apply for a dedicated rehabilitation nursing research position that may benefit the profile and ongoing operation of the program.

\section{Conclusion}

Nurses can generate fresh research insights for evidencebased practice in clinical rehabilitation settings because they have the most contact with the hospital's most important customers - patients. This paper defines and discusses the crucial early steps in developing a RCB program for direct-care rehabilitation nurses with limited resources. This may encourage others to develop and lead research grounded in their clinical practice environment. The process was a learning experience for the team. In particular, they gained skills in developing and conducting a relevant RCB program for direct-care nurses. Working as a team on the program development reinforced the quality of their networking and combined productivity. It was rewarding and energising to see direct-care nurses develop and use their research skills for themselves and for their colleagues, and a tribute to what nurses can achieve in alignment with the strategic goals of a health service.

\section{Acknowledgements}

We thank Metro South Health and The Hopkins Centre for their support. We also thank our participants without whom the RaMP-N would not have been possible. 


\section{Conflict of interest}

The authors declare no conflicts of interest.

\section{Source of funding}

This research was funded in-kind by The Hopkins Centre, the Division of Rehabilitation and the Division of Medicine at Metro South Health Service, Queensland Health.

\section{Ethics}

Ethical approvals for this study were obtained from Griffith University Human Research Ethics Committee (2018/481), and Metro South Health Human Research Ethics Committee (HREC/18/QPAH/271).

\section{References}

Berthelsen, C. (2020). A lack of allocated research time challenges the extent of the implementation of evidence-based practice - A threeyear retrospective follow-up cohort study of Master of Science in Nursing graduates. Journal of Nursing Education and Practice, 10(8), 19-29. doi:10.5430/jnep.v10n8p19

Boehm, B. (1988). A spiral model of software development and enhancement. IEEE Computer, 21(5), 61-72. doi:10.1109/2.59

Brauer, S., Haines, T., \& Bew, P. (2007). Fostering clinician-led research. Australian Journal of Physiotherapy, 53, 143-144.

Chapman, R., Duggan, R., \& Combs, S. (2011). Leading change and advancing health by enhancing nurses' and midwives' knowledge, ability and confidence to conduct research through a clinical scholar program in western Australia. ISRN Nursing, Article ID 245417. doi: $10.5402 / 2011 / 245417$

Cooke, J. (2005). A framework to evaluate research capacity building in health care. BMC Family Practice, 6, 44. doi:10.1186/1471-22966-44

Digby, R., Bolster, D., Perta, A., \& Bucknall, T. (2018). The perspective of allied health staff on the role of nurses in sub acute care. Journal of Clinical Nursing, 27(21-22):4089-4099. doi:10.1111/jocn.14553.

English, R. (2016). Evidence-based teaching tactics for frontline staff using the Clinical Nurse Scholar Model. Journal for Nurse Practitioners, 12(1), e1-5.

Gee, M., \& Cooke, J. (2018). How do NHS organisations plan research capacity development? Strategies, strengths, and opportunities for improvement. BMC Health Services Research, 18(1), 198. doi:10.1186/s12913-018-2992-2

Germov, J. (2005). Theorising health: major theoretical perspectives in health sociology. In J. Germov (Ed.), Second opinion: An introduction to health sociology (3rd ed.). Melbourne, Australia: Oxford University Press.

Greenhalgh, T., MacFarlane, F., Barton-Sweeney, C., \& Woodard, F. (2012). "If we build it, will it stay?" A case study of the sustainability of whole-system change in London. The Milbank Quarterly, 90(3), 516-547. doi:10.1111/j.1468-0009.2012.00673.x

Hagan, J. (2018). Nurse satisfaction with opportunities to engage in research. Western Journal of Nursing Research, 40(2), 209-221. doi:10.1177/0193945916682472

Haywood, H., Pain, H., Ryan, S., \& Adams, J. (2012). Engagement with continuing professional development: Development of a service model. Journal of Allied Health, 41(2), 83-89.
Kahwa, E., Edwards, N., \& Mortley, N. (2016). Research capacity building: A literature review and the theoretical framework. In N. Edwards, D. Kaseje, \& E. Kahwa (Eds.), Building and evaluating research capacity in healthcare systems case studies and innovative models (pp. 11-38). Capetown, South Africa: UCT Press.

Kroehnert, G. (2010). Basic training for trainers: A handbook for new trainers (3rd ed.). North Ryde: McGraw-Hill Australia.

Larson, E., Cohen, B., Gebbie, K., Clock, S., \& Saiman, L. (2011). Interdisciplinary research training in a school of nursing. Nursing Outlook, 59(29-36). doi:10.1016/j.outlook.2010.11.002

Lode, K., Sørensen, E., Salmela, S., A-L, H., \& Severinsson, E. (2015). Clinical nurses' research capacity building in practice: A systematic review. Open Journal of Nursing, 5(7), 664-677. doi:10.4236/ ojn.2015.57070

Marshall, A., Roberts, S., Baker, M., Keijzers, G., Young, J., Stapelberg, N., \& Crilly, J. (2016). Survey of research activity among multidisciplinary health professionals. Australian Health Review, 40(6), 667-673. doi:10.1071/AH15156

McKee, G., Codd, M., Dempsey, O., Gallagher, P., \& Comiskey, C. (2017). Describing the implementation of an innovative intervention and evaluating its effectiveness in increasing research capacity of advanced clinical nurses: Using the consolidated framework for implementation research. BMC Nursing, 16, 21. doi:10.1186/ s12912-017-0214-6

Morris, T. (2019). Experiential learning - A systematic review and revision of Kolb's model. Interactive Learning Environments, 1-4. doi:10.10 80/10494820.2019.1570279

Mundra, S. (2018). Enterprise agility: Being agile in a changing world. Birmingham, Mumbai: Packt Publishing Ltd.

O'Byrne, L., \& Smith, S. (2011). Models to enhance research capacity and capability in clinical nurses: A narrative review. Journal of Clinical Nursing, 2O(9-10), 1365-1371. doi:10.1111/j.13652702.2010.03282.x

O'Regan, G. (2018). The innovation in computing companion. Cham, Switzerland: Springer.

Paget, S., Lilischkis, K., Morrow, A., \& Caldwell, P. (2014). Embedding research in clinical practice: Differences in attitudes to research participation among clinicians in a tertiary teaching hospital. Internal Medicine Journal, 44(1), 86-89. doi:10.1111/imj.12330

Queensland Health. (2020). Magnet hospital recognition. Retrieved from https://metrosouth.health.qld.gov.au/princess-alexandra-hospital/ about-us/magnet-hospital-recognition

Ruparelia, N. (2010). Software development lifecycle models. ACM SIGSOFT Software Engineering Notes, 35(3), 8-13. doi:10.1145/1764810.1764814

Schippers, M., West, M., \& Dawson, J. (2015). Team reflexivity and innovation: The moderating role of team context. Journal of Management, 41(3), 769-788. doi:10.1177/0149206312441210

Trytten, C., Wale, M., Hayes, M., \& Holmes, B. (2019). Lessons learned from a health authority research capacity-building initiative. Healthcare Management Forum, 32(5), 259-265. doi:10.1177/0840470419849468 